\title{
Tecnoestrés y adicción a las tecnologías de la información y las comunicaciones (TIC) en universitarios mexicanos: diagnóstico y validación de instrumento
}

\author{
Armando Sánchez-Macías $^{{ }^{\star} \text {, }}$ Isabel C Flores-Rueda ${ }^{2}$, María G Veytia-Bucheli ${ }^{3}$ y Virginia Azuara-Pugliese ${ }^{1}$ \\ (1) Coordinación Académica Región Altiplano Oeste, Universidad Autónoma de San Luis Potosí, México. \\ (correo-e: armando.sanchez@uaslp.mx; virginia.azuara@uaslp.mx) \\ (2) Coordinación Académica Región Altiplano, Universidad Autónoma de San Luis Potosí, México. \\ (correo-e: isabel.flores@uaslp.mx) \\ (3) Facultad de Ciencias de la Educación, Universidad Autónoma del Estado de Hidalgo, México. \\ (correo-e: maria_veytia@uaeh.edu.mx) \\ * Autor a quien debe ser dirigida la correspondencia.
}

Recibido Feb. 4, 2021; Aceptado Abr. 1, 2021; Versión final Abr. 19, 2021, Publicado Ago. 2021

\begin{abstract}
Resumen
El objetivo de este estudio es identificar y caracterizar en estudiantes de licenciatura el grado de aceptación, el nivel de tecnoestrés y la adicción por el uso de las tecnologías de la información y las comunicaciones (TIC) en procesos académicos. Esta es una investigación cuantitativa con enfoque mixto, de alcance correlacionalexplicativo, no experimental-transversal. El muestreo es no probabilístico por conveniencia a 706 estudiantes de universidades públicas mexicanas. Se utilizó la encuesta de estrés tecnológico de López-Barbosa. Se encontraron bajos niveles de estrés en dos de las poblaciones, otra reportó niveles tendientes a ser altos. El estrés se relaciona con el tiempo de uso de las TIC. Mediante la técnica CB-SEM se encontró que tanto una mala actitud hacia las TIC, así como la adicción, se relacionan de manera causal directa con el tecnoestrés. Se concluye que es indispensable reconocer que las TIC tienen la potencialidad de producir efectos tanto positivos como negativos en profesores y estudiantes.
\end{abstract}

Palabras clave: tecnoestrés; adicción; tecnologías; TIC; estudiante universitario; educación superior

\section{Techno-stress and addiction to information and communication technologies (ICT) in Mexican university students: instrument diagnosis and validation}

\begin{abstract}
The main objective of this study is to identify and characterize the acceptance degree, techno-stress level, and addiction level to information and communication technologies (ICT) of undergraduate students during their studies. This is a mixed-approach quantitative research study. The study's scope is correlational, explanatory, non-experimental, and transversal. The sampling is non-probabilistic for convenience and consists of 706 Mexican public university students. The López-Barbosa technological stress survey is applied. The results show low stress levels in two sub-samples and high stress levels in one sub-sample. Stress is related to ICT time usage. Both bad attitude towards ICT and ICT addiction are directly causally related to techno-stress as shown by using the CB-SEM technique. It is concluded that it is crucial to acknowledge that ICT have the potential to cause positive and negative effects in both professors and students.
\end{abstract}

Keywords: technostress; technology addiction; ICT; college student; higher education 


\section{INTRODUCCIÓN}

La palabra estrés suele tener múltiples significados, suele llamarse estrés al agente, estímulo factor que lo provoca, también a la respuesta biológica al estímulo o también para expresar la respuesta psicológica al estresor. Así mismo, expresa la respuesta psicológica al estresor (Wang, et al., 2020). También se le denominan a las enfermedades psíquicas o corporales provocadas por el estrés, y finalmente, se define como el tema general que puede incluir la diversidad de asuntos que tienen que ver con esta materia. Al hablar del estrés, es pertinente considerar qué estímulos lo producen, cuáles son estos agentes estresantes que lo detonan, y en la actualidad, uno de ellos es sin duda el empleo de las Tecnologías de la Información y la Comunicación (TIC) (Qi, 2019). Por una parte, su uso ha permitido reducir los tiempos para realizar distintas tareas en los diferentes ámbitos del ser humano, así como acortar distancias y favorecer la comunicación tanto de manera sincrónica y asincrónica. Pero a su vez, ha incrementado el tiempo en que los sujetos utilizan las TIC, afectando los niveles de estrés, si por alguna razón no pueden tener conectividad, y manifestando en algunos casos adicción a las mismas. Al profundizar el tema del estrés de manera particular con el empleo de las TIC, se remite al concepto de tecnoestrés, el cual se refiere a una enfermedad de adaptación causada por la falta de habilidad para tratar con las TIC de manera saludable (Hsiao, et al., 2017; Villavicencio-Ayub, et al., 2020).

Además del tecnoestrés, existen diferentes términos que se relacionan con el impacto negativo que se tiene hacia las TIC, entre los que se encuentran: el síndrome de la fatiga informativa (sobrecarga informativa cuando se utilizar el internet), la tecnoadicción (incontrolable compulsión a utilizar las TIC en todo momento y en todo lugar, por largos periodos de tiempo), la tecnofobia (miedo y ansiedad que produce el empleo de las TIC), por mencionar algunos. El empleo de las TIC se ha incrementado en todos los ámbitos de la vida del ser humano, y en el ámbito educativo el incremento es indudable, lo que ha permitido por una parte fortalecer la comunicación, así como innovar en procesos de enseñanza-aprendizaje (Poveda-Pineda y Cifuentes-Medina, 2020), pero a su vez, también están presentes una serie de situaciones que han provocado un crecimiento en el estrés de los estudiantes, así como el desarrollo de algunas fobias. Además de que permanecen creencias y concepciones negativas al respecto de su pertinencia en el desarrollo de procesos pedagógicos (Arancibia, et al., 2020).

Al respecto Upadhyaya y Vrinda (2020) afirman que existe una relación negativa entre el tecnoestrés y la capacidad de ser productivo en la vida académica. Es necesario reflexionar ante la inminente y obligada incorporación de las TIC a los espacios de formación académica. Dado que las TIC no pueden convertirse en una imitación de los espacios presenciales, es necesario estudiar cómo llevar a cabo una incorporación exitosa. Es por ello, que se ha considerado indispensable el estudio del papel del docente y los estudiantes ante la incorporación de las TIC en sus espacios de formación, tales como: actitudes, intereses, predisposición al uso, las dificultades en el uso, interacción entre actores y los escenarios educativos (Poveda-Pineda y Cifuentes-Medina, 2020). Se evidencia que el análisis hacia los docentes ha sido abordado en mayor medida que la de los estudiantes, sin embargo, dado el indisoluble binomio profesor-estudiante, analizar las actitudes y comportamientos de unos, lleva implícito el de los otros.

\section{OTROS ANTECEDENTES}

Hay una serie de antecedentes adicionales que es necesario detallar para documentar en mejor forma este trabajo: i) Actitudes hacia el uso de las TIC en procesos educativos; ii) Aceptación y/o rechazo al uso de las TIC; iii) Niveles de estrés con el uso de las TIC; iv) Adicción en el uso de las TIC; y v) Niveles de uso de las TIC en el ámbito académico.

\section{Actitudes hacia el uso de las TIC en procesos educativos}

En los últimos años, es posible encontrar un incremento en estudios que abordan las actitudes como causa fundamental de la falta de impacto de las TIC en la mejora de resultados educativos (Galván y López, 2017). Es relevante mencionar que el sistema actitudinal se define en tres categorías de componentes conceptuales; afectivo (p.e. sentimientos positivos y/o negativos hacia...), conductual (acciones) y cognitivo (pensamientos). En su conjunto construyen la definición de actitud que se expresa como la consistencia del decir, pensar y actuar en ciertas conductas que pueden predecir otras a futuro (Arancibia, et al., 2020). Otros estudios (Galván y López, 2017) relacionados con la actitud hacia las TIC pretender anticipar el nivel de compatibilidad e involucramiento en proyectos de innovación educativa a través del uso de TIC. Se observa que en conjunto con variables demográficas (sexo, edad) y características docentes (experiencia, escalafón, disponibilidad de laboratorios y equipo de cómputo), existen tres posturas (favorable, neutra y dual) en las actitudes para la incorporación del uso de las TIC en procesos educativos (Arancibia, et al., 2020).

En el estudio de San Nicolás, et al. (2013) se incorpora el desarrollo de actitudes en su manifestación conductual en la dimensión axiológica vinculada al concepto de alfabetización digital. Los resultados demuestran una conducta al desarrollo de tareas tradicionales como; consulta de correo (100\%), uso de 
paquetes ofimáticos (91.27\%), búsqueda de información (96.6\%) y el acceso a aulas virtuales del campus virtual $(96.61 \%)$. Contradictoriamente, muestran bajo uso en redes sociales $(5.82 \%)$, por su parte los estudiantes muestran alto uso (67.91\%). En el contexto empírico cuantitativo Galván y López (2017) afirman que los profesores no parecen estar a favor del uso excesivo de las computadoras en el aula de idiomas porque consideran interrumpen su dinámica y planificación docente. Consideran que el sistema educativo es responsable de que la planificación se dé hacia contextos presenciales, dadas las creencias de que el papel de la computadora en el espacio de formación se limita a juego; entretenimiento y diversión para el alumno, por ello se considera como un premio o recompensa y no como una herramienta pedagógica.

\section{Aceptación y/o rechazo al uso de las TIC}

La búsqueda de las actitudes del profesorado y estudiantes con respecto al uso de las TIC incorporadas a procesos académicos proporciona información con respecto a la posibilidad de adaptar las TIC hacia la construcción de Entornos Virtuales de Aprendizaje. Para ello, investigaciones como la de Rivera-Laylle, et al. (2017) destacan al conocimiento, la actitud y practicidad en la aceptación tecnológica en el ámbito universitario. Dentro de los hallazgos sobresale el hecho de los profesores nunca busquen asesoría de personal experto lo que se presume como una contradicción al aceptar un cambio inminente al uso de las TIC y una renuencia al incorporarlas mientras imparte su materia. Esto incluye el apoyo de sus propios estudiantes o colegas. Las herramientas mayormente utilizadas son: Powerpoint, Word y correo electrónico.

En distintos estudios (Rivera-Laylle, et al., 2017; Sánchez-López, et al., 2014) existe una alta intencióndisposición al uso de las TIC y el profesorado percibe el uso de las TIC como inminente factor de cambio en el proceso educativo, sin embargo, en la práctica no se había generado una necesidad de incorporar e utilizar otro tipo de tecnologías debido a que se consideran como difíciles de utilizar y/o no buscan asesoría externa. El fenómeno expresado en líneas anteriores se documenta teóricamente por Poveda-Pineda y CifuentesMedina, (2020) al enfatizar que, para llevar a cabo una pedagogía flexible particularmente vinculada con TIC -no complementaria sino virtual o semipresencial- es necesario acortar la brecha entre el exceso de confianza del profesorado, estudiantes e IES referente a los contenidos; además se sugiere interpretar la calidad de la educación semipresencial y virtual con precaución y no solamente desde una perspectiva utilitarista como se ha argumentado.

\section{Niveles de estrés con el uso de las TIC}

El impacto de las TIC en profesores y estudiantes se presume es positivo como se ha revisado en las investigaciones anteriores. Sin embargo, es necesario identificar también el impacto negativo ante el uso e implementación de las TIC tanto en estudiantes como en profesores. Al involucrar de forma rápida las TIC en el trabajo académico, pueden fomentar dificultad, preocupación y estrés al diseñar y planificar contenidos (Guzmán, et al., 2011). En el estudio de Guzmán et al. (2011) se concluye que existe interés de incluir herramientas TIC en las aulas, pero los docentes se llegan a frustrar y desmotivar por la dificultad al acceder y manipular las TIC. Por su parte, Rivera-Laylle, et al. (2017), reportan que la desmotivación y frustración conduce a una baja implementación de las TIC en los espacios de formación. Además, de la existencia de poco tiempo por parte del profesorado para preparase en el uso de las herramientas web 2.0. El estrés hacia la incorporación de las TIC a los espacios de formación sugiere que en docentes supone que implica mayor tiempo desde la planificación hasta la impartición de clases, aunque estudios demuestran que los docentes difieren en la importancia de este factor, tales como pertencer a una institución privada (Tapasco y Giraldo, et al., 2017).

Otros estudios identifican que el estrés en los docentes puede surgir a medida que se perciben las TIC como una exigencia a realizar cambios en su dinámica de enseñanza-aprendizaje y que los llevará a cambiar eventual o súbitamente sus rutinas. Incluso se identifica que el estrés puede estar acompañado de ansiedad y actitudes negativas. El estrés y la ansiedad vinculada con el uso de las TIC en ocasiones se presenta por inseguridad e inestabilidad al suponer que se romperán sus rutinas de enseñanza-aprendizaje que llevan años adecuando y dominando por lo que se documentan sensaciones de pérdida de control (Cuadrado, et al., 2009). Otros autores como Degoy y Luque (2013) consideran a las TIC como herramientas susceptibles de desarrollar deseo patológico, frustración, riesgo de aislamiento y uso excesivo acompañado de quejas sociales; que como consecuencia generan un efecto dependencia posiblemente considerado como "un subtipo de adicción comportamental" (p. 2).

\section{Adicción en el uso de las TIC}

El concepto sobre adicción a las TIC no tiene un consenso dentro de las ciencias ni en lo académico, como consecuencia no se observa dentro del manual de diagnóstico de enfermedades y trastornos mentales de la American Psychiatric Association. Autores como Degoy y Luque (2013) refieren a la tecno-dependencia como un patrón desadaptativo de nivel conductual aplicado para la tecnología en general y considera el estudio del uso compulsivo e indiscriminado de TIC. Sin embargo, el fenómeno de uso desmedido de las TIC se observa 
en el contexto donde se dificulta establecer límites entre un buen y mal uso, de manera que es comprensible que el usuario considere una ventaja tener acceso ilimitado a dispositivos electrónicos, internet, contenido audiovisual, entre otros desarrollando una cotidianidad en el comportamiento. Además, la adicción en el uso de las TIC generalmente se encuentra estudiado en el comportamiento de estudiantes y se espera que el docente tenga un rol cooperativo en la prevención de usos abusivos y patológicos de las TIC en alumnos (Degoy y Luque, 2013; Díaz-López, et al., 2020). Es importante reconocer que, existen pocos estudios que revisan la posibilidad que los estudiantes puedan presentar conductas patológicas referentes al uso de las TIC, es decir se considera posible encontrar un vacío teórico con respecto al estudio sobre la adicción a las TIC en procesos académicos.

\section{Niveles de uso de las TIC en el ámbito académico}

En cuanto a la implementación de las TIC por parte del docente, Arancibia, et al., (2020) consideran que la dificultad en la implementación se da cuando el profesor no se siente formado en la aplicación de las TIC en su proceso de enseñanza e implementación en su planeación docente. Algunas de las causas se señalan como: falta de recursos en los laboratorios, limitación en la formación profesional del profesor, actitud de desconfianza (p.e. temor al manejo), tradicionalismo, estructura organizativa, entre otros. Por su parte, CerdaDiaz (2017) destaca que el desarrollo de habilidades digitales es tema central en la educación superior y destaca una competencia transversal de toda la población de las IES. Entre los resultados se comenta que los estudiantes muestran una buena habilidad de TIC, mientras que las IES se esfuerzan por brindar opciones de capacitación de calidad en TIC para los docentes al sentirse abrumadas por las expectativas de los estudiantes chilenos. No obstante, se denotan desempeños favorables en docentes que pertenecen a una generación tecnonativa con experiencia temprana en las TIC.

Finalmente se menciona que en dimensiones sobre Gestión Tecnológica Operativa es necesario considerar las habilidades para el uso de gráficos estadísticos y de Excel. El empleo didáctico de las TIC depende de los niveles de competencias digitales. Lo anterior, no es aplicable en todos los contextos como se demuestra en el estudio de San Nicolás, et al. (2013) en donde se señala un nivel adecuado en el dominio de la TIC por parte de los profesores, sin embargo se visualizan brechas en el uso y la incorporación de desempeños técnicos como la edición de recursos audiovisuales, espacios web 2.0, redes sociales, foros, entre otros. Otro caso favorable, se presenta en el campo de la la educación flexible, el estudio de Vargas, et al., (2010) expone la incorporación de laboratorios virtuales en línea y asincrónicos que tienen como finalidad la experimentación web de los estudiantes en materias de Ingeniería en Sistemas y Automatización. Desde el punto de vista de los estudiantes, se considera un elevado grado de satisfacción, aunque aún presenta desafíos de comunicación con instructores virtuales o la aplicación de Realidad Aumentada. Finalmente, Jiménez, et al. (2020) abordan el uso de las redes sociales entre alumnos y profesores. Los alumnos universitarios realizan un uso intensivo de las redes sociales y forman parte de su vida, en cambio algunos de los profesores se resisten a su uso y aplicación como herramienta de enseñanza-aprendizaje. En el estudio se describe que el uso de las redes sociales comienza a partir de la iniciativa de los estudiantes, lo anterior se señala como posible consecuencia del desfase generacional entre nativos digitales (alumnos) y los migrantes digitales (profesores). También se afirma que se constituyen en una herramienta que permite mejorar el rendimiento académico.

En el contexto actual, a más de un año de que las clases presenciales fueron suspendidas por la pandemia del SARS-COV2 y se trasladaron -mediante el uso intensivo de las TIC- hacia la educación en línea, virtual, a distancia, o una combinación de éstas, se considera de suma importancia identificar el impacto que puede tener en el alumnado. Por lo tanto, el objetivo de esta investigación es identificar en estudiantes de tres entidades de nivel Licenciatura el grado de aceptación, el nivel de estrés y la adicción por el uso de de las TIC en procesos académicos, así como caracterizar las relaciones entre estas variables.

\section{METODOLOGÍA}

Es una investigación cuantitativa con enfoque mixto; de alcance correlacional-explicativo, no experimental y transversal. La población son estudiantes de Licenciatura de tres entidades mexicanas de nivel superior: a) la Coordinación Académica Región Altiplano Oeste (CARAO) de la Universidad Autónoma de San Luis Potosí en Salinas, San Luis Potosí; b) la Facultad de Ciencias Económicas y Administrativas de la Universidad Autónoma del Carmen (UNACAR) en Ciudad del Carmen, Campeche y; c) el Instituto de Ciencias Sociales y Humanidades y de la Universidad Autónoma del Estado de Hidalgo (UAEH) en Pachuca de Soto, Hidalgo. Se utilizó un muestreo de tipo no probabilístico en la población estudiantil de dichos centros universitarios con un tamaño de 706 estudiantes, en todos los casos se aplicó a más del $90 \%$ de la población inscrita en la facultad o escuela. La muestra se distribuye de la siguiente forma: CARAO, 230 (32.6\%); UNACAR, 252 (35.7\%); UAEH, 224 (31.7\%).

Se utilizó el cuestionario Encuesta de estrés tecnológico de López-Barbosa que mide cuatro dimensiones en relación con uso de las TIC, a saber: (1) Actitudes de aceptación o rechazo del uso de TIC que se manifiestan 
en comportamientos que influyen en el proceso educativo denominado Actitud Frente a las TIC (AFT) (Arancibia, et al., 2020); (2) Niveles de estrés por el uso de TIC denominado Estrés Escolar (ELE). Se refiere al grado de estrés derivado del uso de las TIC, denominado como tecnoestrés (Qi, 2019; Villavicencio-Ayub, et al., 2020; Wang, et al., 2020); (3) niveles de adicción en el uso de las TIC denominado Efectos del Uso de las TIC (EUTIC). Se refiere a la adicción en el uso de las TIC (Nelson y Pieper, 2020); (4) niveles de uso de las TIC en el ámbito académico que a su vez considera las TIC de forma global y específicamente el uso de redes sociales, se denomina Redes Sociales y TIC en Educación (RSYTIC) (Cerda-Diaz, 2017; San Nicolás et al., 2013; Vargas, et al., 2010). El cuestionario incluye una sección de aspectos sociodemográficos de los participantes para después realizar una exploración de las cuatro dimensiones mediante una escala cuantitativa de tipo Likert que va de 5 hasta 1 como sigue: completamente de acuerdo; de acuerdo, más o menos de acuerdo; en desacuerdo y completamente en desacuerdo. En cuanto a las dimensiones, valores de dos o menores se consideran bajos, mientras que valores de cuatro y mayores se consideran altos. Las hipótesis planteadas son: $\mathrm{H}_{1}$ El Estrés Escolar (ELE) se relaciona de manera estadísticamente significativa con el tiempo utilizando las TIC; $\mathrm{H}_{2}$ Los Efectos del Uso de TIC (EUTIC) (adicción) son causa del Estrés Escolar (ELE); $\mathrm{H}_{3}$ Una mala Actitud frente a las TIC (AFT) son causa del Estrés Escolar (ELE) y; $\mathrm{H}_{4} \mathrm{El}$ uso de las TIC en procesos educativos (RSYTIC) son causa del Estrés Escolar (ELE). Para identificar relaciones estadísticamente significativas entre las dimensiones se utilizó la prueba $\mathrm{H}$ de Kruskal-Wallis para muestras independientes, así como la prueba de correlación no paramétrica rho de Spearman.

\section{RESULTADOS}

La consistencia interna del instrumento se calculó mediante el estadístico Alpha de Cronbach por dimensión y luego por mitades. En las cuatro dimensiones se obtuvieron valores aceptables (ver tabla 1 y 2).

Tabla 1: Resultado Alpha de Cronbach

\begin{tabular}{|l|c|c|c|c|}
\hline \multicolumn{1}{|c|}{ Dimensión } & $\begin{array}{c}\text { Número de } \\
\text { ítems inicial }\end{array}$ & $\begin{array}{c}\text { Alpha de } \\
\text { Cronbach }\end{array}$ & $\begin{array}{c}\text { Número de } \\
\text { ítems final }\end{array}$ & $\begin{array}{c}\text { Alpha de } \\
\text { Cronbach }\end{array}$ \\
\hline Actitud frente a las TIC (AFT) & 20 & 0.846 & 15 & 0.906 \\
\hline Estrés escolar (ELE) & 10 & 0.682 & 7 & 0.768 \\
\hline Efectos del uso de TIC (EUTIC) & 10 & 0.929 & 10 & 0.929 \\
\hline Redes sociales y TIC en la educación (RSYTIC) & 19 & 0.916 & 19 & 0.916 \\
\hline
\end{tabular}

Tabla 2: Resultado prueba de las dos mitades

\begin{tabular}{|l|c|c|c|c|}
\hline \multicolumn{1}{|c|}{ Dimensión } & $\begin{array}{c}\text { Número de ítems } \\
\text { primera mitad }\end{array}$ & $\begin{array}{c}\text { Alpha de } \\
\text { Cronbach }\end{array}$ & $\begin{array}{c}\text { Número de ítems } \\
\text { segunda mitad }\end{array}$ & $\begin{array}{c}\text { Alpha de } \\
\text { Cronbach }\end{array}$ \\
\hline Actitud frente a las TIC (AFT) & 8 & 0.844 & 7 & 0.856 \\
\hline Estrés escolar (ELE) & 4 & 0.598 & 3 & 0.688 \\
\hline Efectos del uso de TIC (EUTIC) & 5 & 0.858 & 5 & 0.894 \\
\hline Redes sociales y TIC en la educación (RSYTIC) & 10 & 0.891 & 9 & 0.822 \\
\hline
\end{tabular}

Mediante el uso del Análisis Factorial Exploratorio (AFE) se identificaron las variables latentes (factores) a partir de las observadas (ítems). Se utilizó el tipo de extracción de máxima verosimilitud y la rotación ortogonal de tipo varimax con normalización Kaiser para maximizar el valor de las cargas en los factores (Watkins, 2018), dado que es recomendado cuando luego se procede al modelo de Ecuaciones Estructurales Basado en Covarianzas (CB-SEM por sus siglas en inglés) (Hair Jr., et al., 2017). El tamaño de la muestra utilizado $(n=707)$ se considera apropiado dado que existe una proporción de 12:1 en cuanto a elementos de la muestra e ítems en el cuestionario respectivamente (Watkins, 2018). La medida KMO obtuvo un valor de 0.952 , mientras que el coeficiente de esfericidad de Bartlett obtuvo un valor estadísticamente significativo $(p=0.00)$. Dichos resultados confirman la pertinencia del uso del AFE en este trabajo. Como se observa en la tabla 3 se encontró que las cargas factoriales se agrupan razonablemente en cuatro factores, concentrando a sus respectivos indicadores. Por lo cual, se considera que el instrumento es razonablemente apropiado para medir la variable. Aquellos indicadores que no alcanzaron una carga factorial de al menos 0.3 fueron eliminados. Se observa que tres factores tienen al menos diez indicadores. En el caso del factor que se refiere al estrés escolar (ELE) se obtuvieron únicamente tres indicadores, la cual se considera un nivel aceptable de acuerdo con la literatura (Watkins, 2018). Se encontraron cuatro factores que explican el $53.21 \%$ de la varianza total.

En cuanto a las características sociodemográficas más releventes se tiene que $58.8 \%$ de los encuestados son mujeres, el $40.5 \%$ hombres y el resto se identificó como otro. La media de las edades es de 19.9 años $(\mathrm{s}=1.78)$. La media del semestre que cursan es $3.5(\mathrm{~s}=2.38)$. En cuanto al tiempo que pasan utilizando las TIC se encontró lo siguiente: de 1 a 4 horas al día el 33.5\%, entre 5 y 8 horas al día $41.7 \%$, entre 9 y 12 horas $17.7 \%$ y más de 12 horas $5.9 \%$. Cabe destacar que un mayor de 4 horas se considera alto e incluye al $66.5 \%$ del total. En cuanto a los resultados descriptivos encontrados en las dimensiones estudiadas se encontraron los valores que se muestran en la tabla 4. 
Tabla 3: Matriz factorial

\begin{tabular}{|c|c|c|c|c|}
\hline \multirow{2}{*}{ Indicador } & \multicolumn{4}{|c|}{ Factor } \\
\hline & 1 & 2 & 3 & 4 \\
\hline AFT 1 & & & .381 & \\
\hline AFT 4 & & & .408 & \\
\hline AFT 5 & & & .603 & \\
\hline AFT 6 & & & .631 & \\
\hline AFT 8 & & & .565 & \\
\hline AFT 9 & & & .737 & \\
\hline AFT 10 & & & .752 & \\
\hline AFT 11 & & & .691 & \\
\hline AFT 12 & & & .531 & \\
\hline AFT 13 & & & .392 & \\
\hline AFT 14 & & & .536 & \\
\hline AFT 15 & & & .606 & \\
\hline AFT 17 & & & .443 & \\
\hline AFT 18 & & & .456 & \\
\hline AFT 20 & & & .378 & \\
\hline ELE 1 & & & & .354 \\
\hline ELE 2 & & & & .543 \\
\hline ELE 8 & & & & .304 \\
\hline EUTIC 1 & & .519 & & \\
\hline EUTIC 2 & & .691 & & \\
\hline EUTIC 3 & & .763 & & \\
\hline EUTIC 4 & & .718 & & \\
\hline EUTIC 5 & & .736 & & \\
\hline EUTIC 6 & & .687 & & \\
\hline EUTIC 7 & & .679 & & \\
\hline EUTIC 8 & & .692 & & \\
\hline EUTIC 9 & & .648 & & \\
\hline EUTIC 10 & & .545 & & \\
\hline RSYTIC 1 & .656 & & & \\
\hline RSYTIC 2 & .745 & & & \\
\hline RSYTIC 3 & .744 & & & \\
\hline RSYTIC 4 & .759 & & & \\
\hline RSYTIC 5 & .783 & & & \\
\hline RSYTIC 6 & .664 & & & \\
\hline RSYTIC 7 & .745 & & & \\
\hline RSYTIC 8 & .677 & & & \\
\hline RSYTIC 10 & .549 & & & \\
\hline RSYTIC 11 & .664 & & & \\
\hline RSYTIC 12 & .713 & & & \\
\hline RSYTIC 13 & .312 & & & \\
\hline RSYTIC 14 & .547 & & & \\
\hline RSYTIC 15 & .742 & & & \\
\hline RSYTIC 16 & .739 & & & \\
\hline RSYTIC 17 & .708 & & & \\
\hline
\end{tabular}

Tabla 4: Resultados descriptivos

\begin{tabular}{|l|c|c|c|c|c|c|c|c|}
\hline & \multicolumn{2}{|c|}{ AFT } & \multicolumn{2}{c|}{ ELE } & \multicolumn{2}{c|}{ EUTIC } & \multicolumn{2}{c|}{ RSYTIC } \\
\cline { 2 - 9 } & Media & Desv est & Media & Desv est & Media & Desv est & Media & Desv est \\
\hline CARAO & 2.4 & 1.0 & 2.2 & 1.0 & 2.1 & 1.1 & 3.6 & 1.1 \\
\hline UNACAR & 3.4 & 1.1 & 3.6 & 1.1 & 3.6 & 1.2 & 2.4 & 1.0 \\
\hline UAEH & 2.5 & 1.1 & 2.2 & 1.2 & 2.2 & 1.2 & 3.7 & 1.2 \\
\hline PROMEDIO & 2.8 & 1.2 & 2.7 & 1.3 & 2.7 & 1.4 & 3.2 & 1.3 \\
\hline
\end{tabular}

Como se observa en la tabla 4, la mayor parte de las medias se encuentra en rangos de entre 2 y 3 , mientras que la desviación estándar oscila en valores un poco mayores a 1, lo cual muestra estabilidad en las respuestas. En cuanto a las dimensiones y sus valores, se encontró lo siguiente: (1) en cuanto a la Actitud Frente a las TIC (AFT), valores altos implican mayor rechazo, valores bajos representan menor rechazo. Se encontraron valores bajos en CARAO y UAEH, mientras que en UNACAR fueron intermedios. En términos generales se considera existen actitudes apropiadas hacia el uso de las TIC, lo cual es favorable para su incorporación en procesos académicos; (2) al respecto del Estrés Escolar (ELE), a mayores valores mayor estrés y a menores valores menor estrés. Se identificaron bajos niveles de estrés en CARAO y UAEH, 
mientras que en UNACAR se considera una tendencia hacia el estrés, lo cual puede manifestarse como frustración, ansiedad y desesperación derivado de utilizar las TIC en procesos académicos; (3) los resultados de los Efectos del Uso de las TIC (EUTIC), mayores niveles manifiestan la existencia de adicción a las TIC y valores más bajos su ausencia a las TIC en los estudiantes. No se considera que exista adicción en la muestra de CARAO y de UAEH, mientras que en UNACAR sí existen niveles aunque moderados de adicción, lo cual podemos relacionar con la presencia de niveles de estrés; (4) en cuanto a las Redes Sociales y TIC en la Educación (RSYTIC), mayores valores manifiestan una mayor implicación de las TIC en procesos educativos. En contraste con las dimensiones anteriores en este caso existe una mayor implicación en CARAO y UAEH, mientras que la UNACAR es aquella muestra que manifiesta menor uso de redes sociales y TIC en Educación.

En la figura 1 se evidencia que dos de las muestras (CARAO y UAEH) tienen similitudes en las variables estudiadas y diferencias claras con la tercera muestra (UNACAR). Cuando los niveles de estrés escolar son mayores viene acompañado de mala actitud hacia las TIC y adicción en el uso de las TIC. Según la escala, el nivel de estrés escolar es bajo en la CARAO y la UAEH, y moderado con tendencia a alto en la UNACAR. Se observa también que existe una relación inversa entre el estrés escolar y el uso de las TIC para fines educativos. Mediante la prueba no paramétrica $\mathrm{H}$ de Kruskal-Wallis para muestras independientes se comprueba que pertenecer a una $u$ otra universidad, se relaciona con las diferencias en las medianas de las variables AFT, ELE, EUTIC y RSYTIC al obtener en todos los casos niveles estadísticamente significativos $(p<0.05)$. Mediante la prueba post hoc se encontró que las diferencias se encuentran entre CARAO y UNACAR $(p<0.05)$ y UAEH y UNACAR $(p<0.05)$.

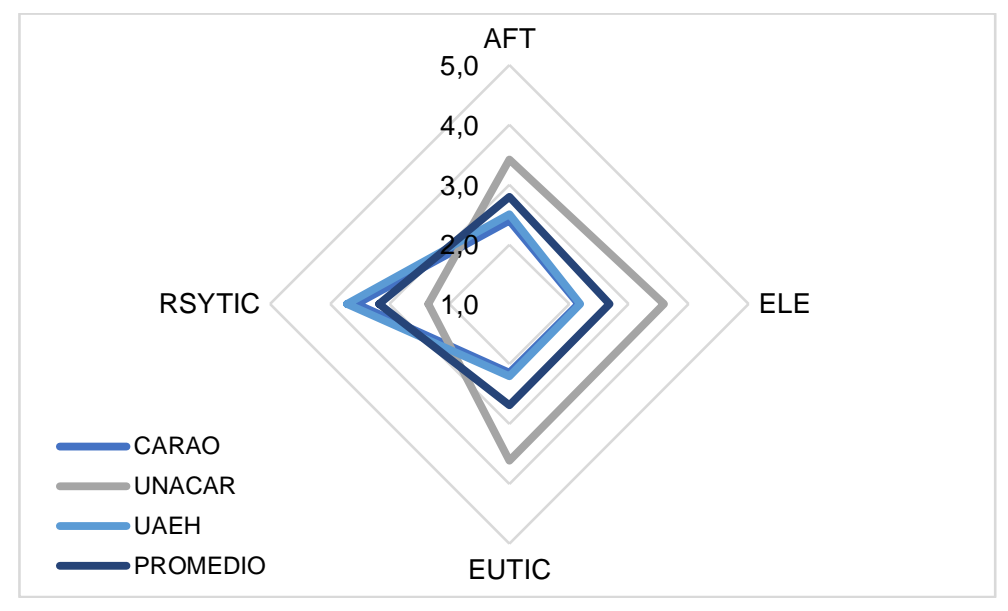

Fig. 1: Resultados descriptivos de los datos analizados por Universidad

No se encontró una relación estadísticamente significativa entre las dimensiones analizadas y las variables: género, número de horas que utilizan las TIC y la edad. Sin embargo, sí se encontró con la variable semestre,

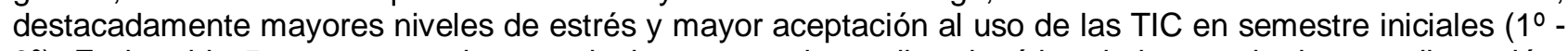
$\left.3^{\circ}\right)$. En la tabla 5 se muestran las correlaciones entre la media aritmética de los resultados por dimensión, cabe destacar que el tipo de correlación (directa o inversa) es consistente en las tres muestras, esto valida las relaciones determinadas previamente al comparar los resultados descriptivos, considerando además que todas las correlaciones en la prueba rho de Spearman resultaron estadísticamente significativas $(p<0.01)$. (Las correlaciones estadísticamente significativas al nivel de 0.01 se muestran con los signos ${ }^{* *}$ ).

Tabla 5: Correlación no paramétrica Rho de Spearman. Datos promedio de las dimensiones estudiadas

\begin{tabular}{|l|l|l|l|l|}
\hline & AFT & ELE & EUTIC & RSYTIC \\
\hline AFT & 1.000 & $.709^{* *}$ & $.600^{* *}$ & $-.393^{* *}$ \\
\hline ELE & $.709^{* *}$ & 1.000 & $.702^{* *}$ & $-.444^{* *}$ \\
\hline EUTIC & $.600^{* *}$ & $.702^{* *}$ & 1.000 & $-.398^{* *}$ \\
\hline RSYTIC & $-.393^{* *}$ & $-.444^{* *}$ & $-.398^{* *}$ & 1.000 \\
\hline
\end{tabular}

El estrés escolar aumenta cuando existen altos niveles de adicción. También se evidencia una correlación inversa aunque débil entre el uso de las TIC para fines educativos y el estrés escolar. Se podría inferir -sin afirmarlo categóricamente- que la familiaridad y habilidad en el uso de las TIC podría disminuir el estrés como sugiere la literatura citada. Por otro lado, cabe destacar que el semestre también se relaciona con las variables AFT, ELE, EUTIC y RSYTIC al obtener niveles estadísticamente significativos $(p<0.05)$ en la prueba $\mathrm{H}$ de Kruskal-Wallis. Considerando el semestre las muestras se comportan de manera similar y que el RSYTIC adopta valores contrarios a la AFT, ELE y EUTIC (ver figura 2). Con la evidencia obtenida se procede a validar las relaciones entre las variables mediante el modelo CB-SEM, por su utilidad para validar teoría (Hair Jr., et al., 2017). 


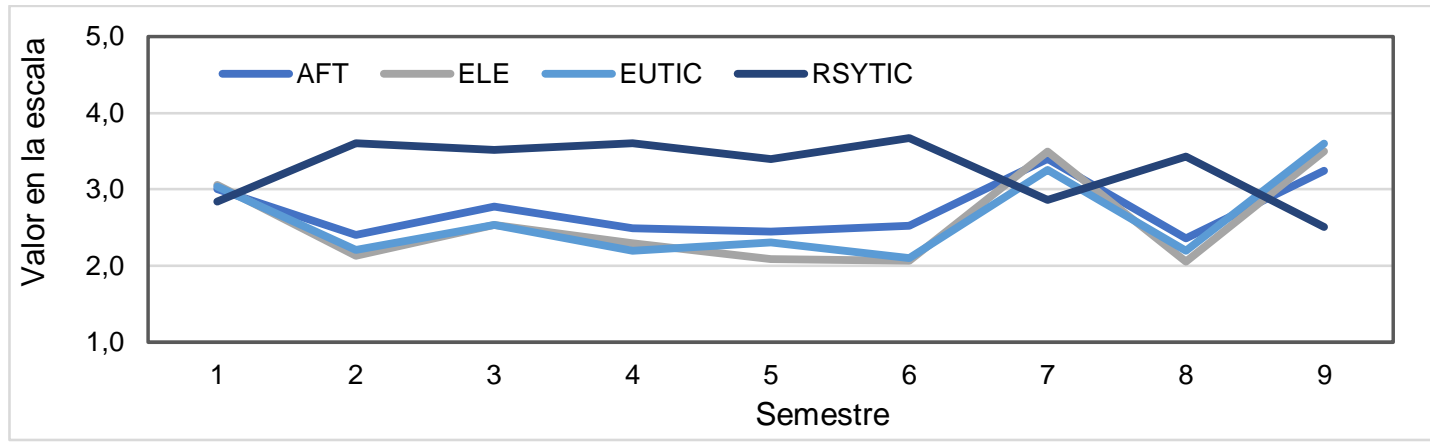

Fig. 2: Resultados descriptivos de los datos analizados por semestre

En cuanto a los parámetros para validar el ajuste del modelo se encontró lo siguiente. La razón $x^{2} / g l$ fue de 2.8713, mientas que en los índices de ajuste global del modelo teórico la raíz cuadrada del error medio cuadrático (RMSEA) obtuvo un valor de 0.074, menor al 0.08 recomendado. El Índice de Ajuste Normalizado (NFI) arrojó un valor de 0.91, un poco superior al mínimo requerido de 0.90 . El Índice de Ajuste Comparativo (CFI) fue de 0.903 , superior al 0.90 que se considera como adecuado. Por su parte el Índice de ajuste normado de parsimonia (PNFI) que relaciona los constructos con la teoría que los sustenta obtuvo un valor de 0.857 , el cual mientras más cerca esté de 1.0 muestra una mayor relación (Hair Jr., et al., 2017). El modelo se presenta en la figura 3.

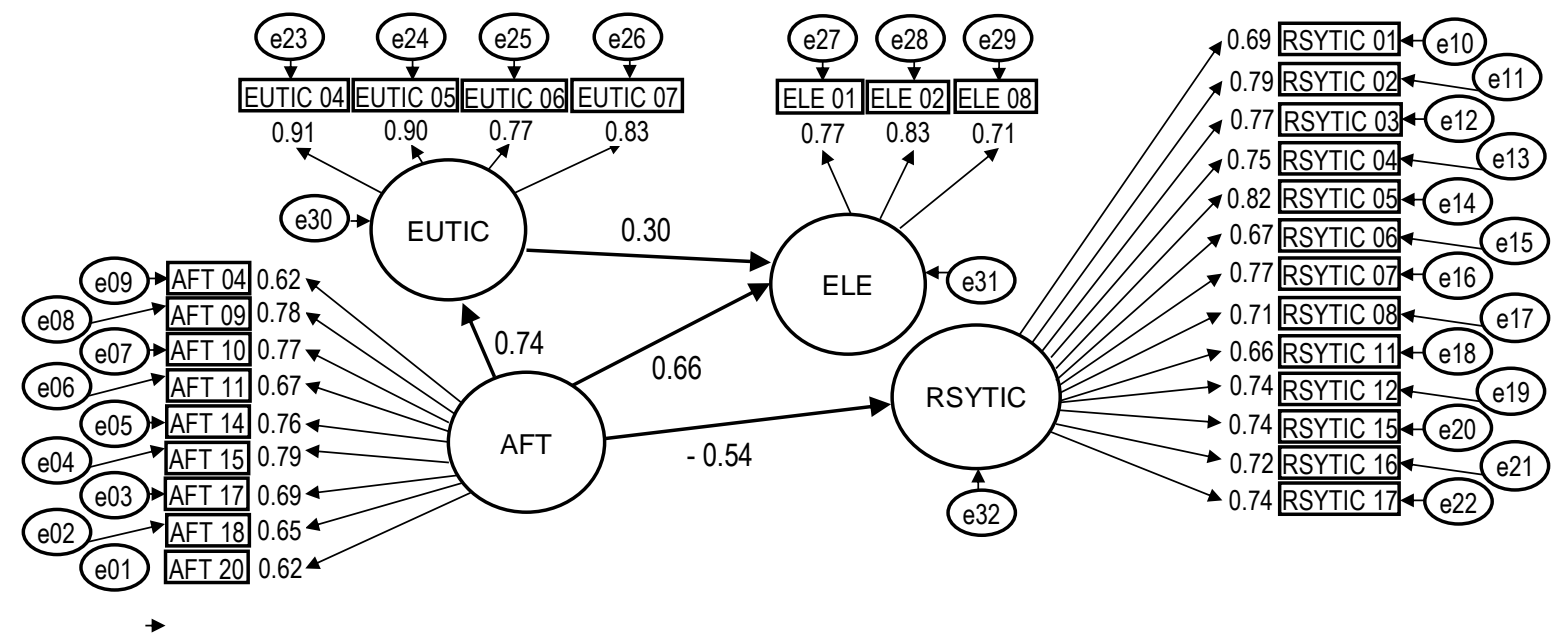

Fig. 3: Modelo de ecuaciones estructurales de las variables estudiadas

Del análisis CB-SEM se presentan los siguientes hallazgos: (a) la adicción a las TIC tiene una relación causal directa hacia el estrés con un coeficiente estimado de 0.30; (b) la actitud frente a las TIC tiene una relación causal directa hacia el estrés con un coeficiente estimado de 0.66; (c) también existe una relación causal indirecta hacia el estrés pero que incluye a la adicción con un coeficiente estimado de $(0.74 \times 0.30) 0.22$ y; (d) finalmente, la actitud frente a las TIC tiene una relación causal directa y negativa hacia el uso de las redes sociales y TIC en procesos educativos.

\section{DISCUSIÓN}

Para Tarafdar, et al. (2019) el tecnoestrés es un fenómeno que se está expandiendo a través de todo tipo de organizaciones, además, existen lagunas teóricas en el campo educativo que requieren ser estudiados desde un enfoque multidisciplinario. Es un fenómeno que debe analizarse desde una perspectiva multidisciplinaria dadas sus caracaterísticas y su relación con la adicción a la tecnología (Tarafdar, et al., 2020). Para esta investigación se considera el análisis de factores en los que se involucra el uso de las TIC, en específico su uso en procesos educativos, la actitud y la adicción. La investigación empírica reveló niveles bajos de estrés en la muestra de CARAO y de la UAEH, más elevados con tendencia a ser altos en la UNACAR. Esto coincide por lo reportado por Hsiao, et al., (2017) en universitrios taiwaneses (un valor de 2.283, cuando valores menores a 3 se consideran bajos). En la muestra no se encontró que el género, el programa académico y la edad se relacionaran con el nivel de estrés; pero sí el semestre cursado, esto contrasta con lo encontrado por Zavala-Romero (2018), quien reporta mayores niveles de estrés en el género femenino del estudiantado universitario de la Ciudad de México. No se encontró una relación estadísticamente significativa entre una mayor presencia de estrés en las personas que pasan más tiempo utilizando las TIC, por lo cual se rechaza 
la primera hipótesis planteada. Se encontró que correlacionan de manera inversa $(-0.444 ; p<0.01)$. Esto coincide con los resultados de Qi (2019) quien afirma que una sobrecarga tecnológica es causante de mayor estrés, además de la denominada tecnoinvasión: cuando la persona puede ser contactada en cualquier lugar y a cualquier hora. Díaz-López, et al. (2020) en adolescentes españoles encontraron también una relación directa y estadísticamente significativa entre la frecuencia de las TIC y los niveles de estrés.

La segunda hipótesis establece que los Efectos del Uso de TIC (EUTIC) (adicción) son causa del Estrés Escolar (ELE). Se acepta, dado que se encontró un relación causal directa con un coeficiente estimado de 0.30. Los resultados confirman lo expuesto por Zavala-Romero (2018) quien encontró que la adicción se asocia al estrés derivado de la "[...] sobrecarga y fatiga cognitiva, debido a la concentración mental y al grado de uso continuo de las TIC" (p.30). Esto conicide con lo que reportado por Tarafdar, et al. (2020), quienes reportaron una correlación directa entre adicción y el estrés mediada por los denominados distractores. En cuanto a la tercera hipótesis que establece que una mala Actitud frente a las TIC (AFT) es causa del Estrés Escolar (ELE) se acepta, dado que se encontró una relación causal directa. Marchiori, et al. (2018) concuerdan con esta relación, y agregan que existen factores sociodemográficos que condicionan la aceptación a las TIC y que, a su vez, dan pie a la prevalencia de diversas manifestaciones del tecnoestrés: mayor edad que propicia la tecno-complejidad, ser mujer la tecno-incertidumubre y ser hombre la tecno-invasión. Por otro lado, Revilla, et al. (2016) afirman que también la capacitación en el uso de las TIC permite reducir la tecnoansiedad y el tecnoestrés.

Finalmente, se encontró un relación causal directa y negativa entre malas actitudes (AFT) y el uso de las TIC en procesos educativos (RSYTIC), eso afirma el hecho de que malas actitudes impiden la adopción de las TIC para alcanzar objetivos académicos, en este sentido Prior, et al. (2016) afirman que una actitud positiva y habilidades en el uso de las TIC contribuyen de manera significativa a la autoeficacia, la cual, a su vez tiene efectos positivos en el aprendizaje cooperativo y la interacción dentro de los LMS.

\section{CONCLUSIONES}

La incorporación de las TIC a los procesos educativos se ha constituido como un nuevo paradigma de la acción educativa. En este sentido, se considera indispensable reconocer que tienen la potencialidad de producir efectos tanto positivos como negativos en profesores y estudiantes. Por lo tanto, es necesario que se considere de suma importancia poner atención en las repercusiones indeseables derivadas de su uso cotidiano. Transitar de la inclusión de las TIC, ya no únicamente como una herramienta de apoyo para el proceso enseñanza-aprendizaje, sino como un sistema didáctico-pedagógico, debe considerar las implicaciones contextuales en las que se desenvuelven estudiantes y profesores. El tecnoestrés y la tecnoadicción son una realidad que se consituye como un factor que debe ser tomado en cuenta en los procesos de gestión educativa, a nivel institucional (la escuela) y a nivel pedagógico (el aula). En esta investigación se contribuye con la validación de relaciones teóricas relativas al tecnoestrés y a los procesos educativos. Así mismo, se validó la pertinencia del instrumento utilzado. Se tuvo como principal limitación la posibilidad de utilizar un muestreo más amplio y de tipo probabilístico, sin embargo, el análisis aquí presentado cuenta con la robustez apropiada para ser concluyente con los métodos utilizados. A través de esta investigación se identificó el grado de aceptación, nivel de estrés y la adicción por el uso de las TIC en procesos académicos y se caracterizaron sus relaciones. Los resultados tienen implicaciones para la educación superior: mostrar el impacto de factores como la adicción y la actitud hacia las TIC en el tecnoestrés, considerar sus efectos para la planeación, toma de decisiones y prácticas educativas en directivos y profesores, así como considerar el nuevo contexto en el diseño de los planes y programas que a partir de ahora deberan asumir a las TIC como mediadores indispensables de los procesos pedagógicos.

\section{REFERENCIAS}

Arancibia, M.L., Cabero, J., y Marín, V., Creencias sobre la enseñanza y uso de las tecnologías de la información y la comunicación (TIC) en docentes de educación superior, https://doi.org/10.4067/s0718-50062020000300089, Form. Univ., 13(3), 89-100 (2020).

Cerda-Diaz, L., Assessment in TIC skills in pedagogy students of the Catholic University of Maule (Chile), https://doi.org/10.1016/j.sbspro.2017.02.125, Procedia - Social and Behavioral Sciences, 237, 893-899 (2017).

Cuadrado, I., Fernández, I., y Ramos, J.L., Impacto de las TICs en las Actitudes, Formación y Utilización del Profesorado en el Contexto Escolar Extremeño (1998-2006), Campo Abierto, 28(1), 13-33 (2009).

Degoy, E., y Luque, L.E., El Rol del Docente ante las Adicciones Tecnológicas. ¿Factor de Protección o Riesgo?, https://doi.org/10.35362/rie614931, Revista Iberoamericana de Educación, 61(4), 1-10 (2013).

Díaz-López, A., Maquilón-Sánchez, J., y Mirete-Ruiz, A., Maladaptive use of ICT in Adolescence: Profiles, Supervision and Technological Stress, https://doi.org/10.3916/C64-2020-03, Comunicar, 64, 29-38 (2020).

Galván, C., y López, M., ICT in the English Classroom. Qualitative Analysis of the Attitudes of Teachers of English towards its Implementation in Secondary School, https://doi.org/10.1016/j.sbspro.2017.02.074, Procedia-Social and Behavioral Sciences, 237, 268-273 (2017). 
Guzmán, T., García, M.T., y otros dos autores, Formación Docente para la Integración de las TIC en la Práctica Educativa, Apertura, 3(1), (2011).

Hair Jr, J. F., Babin, B. J., y Krey, N., Covariance-Based Structural Equation Modeling in the Journal of Advertising: Review and Recommendations, https://doi.org/10.1080/00913367.2017.1281777, J. Advertising, 46(1), 163-177 (2017).

Hsiao, K. L., Shu, Y., y Huang, T. C., Exploring the Effect of Compulsive Social App Usage on Technostress and Academic Performance: Perspectives from Personality Traits, https://doi.org/10.1016/j.tele.2016.11.001, Telematics and Informatics, 34(2), 679-690 (2017).

Jiménez, M., Pérez, F. y Gómez, P., Análisis de los factores tecnológicos sobre el rendimiento académico en una universidad pública en la Ciudad de México, http://dx.doi.org/10.4067/S0718-50062020000600255, Form. Univ., 13(6), 255-266 (2020).

Marchiori, D. M., Mainardes, E. W., y Rodrigues, R. G., Do Individual Characteristics Influence the Types of Technostress Reported by Workers?, https://doi.org/10.1080/10447318.2018.1449713, International Journal of HumanComputer Interaction, 1-13 (2018).

Nelson, J. J. y Pieper, C. M., Who's an iAddict? A Sociodemographic Exploration of Device Addiction Among American Adults, https://doi.org/10.1111/ssqu.12833, Social Science Quarterly, 101(5), 2071-2084 (2020).

Padilla-Beltrán, J.E., Vega-Rojas, P.L. y Rincón-Caballero, D.A., Tendencias y Dificultades para el Uso de las TIC en Educación Superior, Entramado, 10(1), 272-295 (2014).

Poveda-Pineda, D.F., y Cifuentes-Medina, J.E, Incorporación de las tecnologías de información y comunicación (TIC) durante el proceso de aprendizaje en la educación superior, http://dx.doi.org/10.4067/S0718-50062020000600095, Form. Univ., 13(6), 95-104 (2020).

Prior, D.D., Mazanov, J. y otros tres autores, Attitude, Digital Literacy and Self Efficacy: Flow-on Effects for Online Learning Behavior, https://doi.org/10.1016/j.iheduc.2016.01.001, The Internet and Higher Education, 29, 91-97 (2016).

Qi, C., A double-edged sword? Exploring the Impact of Students' academic Usage of Mobile Devices on Technostress and Academic Performance, https://doi.org/10.1080/0144929X.2019.1585476, Behaviour \& Information Technology, 38(12), 1-18 (2019).

Revilla, O., Alpiste, F. y otros dos autores, Reducing Techno-anxiety in High School Teachers by Improving their ICT Problem-solving Skills, https://doi.org/10.1080/0144929X.2016.1221462, Behaviour \& Information Technology, 36(3), 255-268 (2016).

Rivera-Laylle, L.I., Fernández-Morales, K., y otros dos autores, La Aceptación de las TIC por Profesorado Universitario: Conocimiento, Actitud y Practicidad, https://doi.org/10.15359/ree.21-3.6, Educare, 21(3),1-18 (2017).

San Nicolás, M.B., Fariña, E. y Area, M., Competencias digitales del profesorado y alumnado en el desarrollo de la docencia virtual. el caso de la Universidad de la Laguna, Rev. Historia de la Educación Latinoamericana, 14(19), (2013).

Sánchez-López, P., Andrés, M.P., y Soriano, M., Opinión de los Docentes y Estudiantes acerca del uso de las TIC como Herramienta para la Inclusión de una Estudiante con Discapacidad. Revista Interuniversitaria de Formación del Profesorado, 28(1), 67-82 (2014).

Tapasco, O.A., y Giraldo, J.A, Estudio Comparativo sobre Percepción y uso de las TIC entre Profesores de Universidades Públicas y Privadas, http://dx.doi.org/10.4067/S0718-50062017000200002, Form. Univ., 10(2),3-12 (2017).

Tarafdar, M., Cooper, C.L., y Stich, J-F. The Technostress Trifecta-techno Eustress, Techno Distress and Design: Theoretical Directions and an Agenda for Research, https://doi.org/10.1111/isj.12169, Info. Systems J., 29(1) 6-42 (2019).

Tarafdar, M., Maier, C. y otros dos autores, Explaining the link between technostress and technology addiction for social networking sites: A study of distraction as a coping behavior, https://doi.org/10.1111/isj.12253, Info. Systems J., 30(1), 96-124 (2020).

Upadhyaya, P. y Vrinda, Impact of Technostress on Academic Productivity of University Students, https://doi.org/10.1007/s10639-020-10319-9, Education and Information Technologies, (2020).

Vargas, H., Sánchez, J. y otros cuatro autores, Docencia en Automática: Aplicación de las TIC a la realización de Actividades Prácticas a través de Internet, https://doi.org/10.1016/S1697-7912(10)70006-6, Revista Iberoamericana de Automática e informática industrial, 7(1), 35-45 (2010).

Villavicencio-Ayub, E., Ibarra, D.G. y Calleja, N., Tecnoestrés en Población mexicana y su Relación con Variables Sociodemográficas y Laborales, https://doi.org/10.17081/psico.23.44.3473, Psicogente, 23(44), (2020).

Wang, X., Tan, S.C., y Li, L., Technostress in University Students' technology-enhanced learning: An Investigation from Multidimensional Person-environment Misfit, https://doi.org/10.1016/j.chb.2019.106208, Computers in Human Behavior, 105,1-36 (2020).

Watkins, M.W., Exploratory Factor Analysis: A Guide to Best Practice, https://doi.org/10.1177/0095798418771807, Journal of Black Psychology, (44)3 219-246 (2018).

Zavala-Romero, E. Á., La Adicción y Ansiedad Vinculadas a las Tecnologías de la Información y Comunicación, Incidencia en la Calidad de Vida de los Estudiantes, Científica, 22(1), (2018). 